\title{
Detecção imuno-histoquímica de células apoptóticas em glioblastoma recidivante de paciente tratado com álcool perílico por via inalatória
}

\author{
Clovis Orlando da Fonseca', Hilda Petrs Silva², Débora Futuro³, Leonardo \\ Miguez, Janaina Nagel ${ }^{5}$, Vinícius Ribas ${ }^{6}$, Rafael Linden ${ }^{7}$, Gilberto \\ Schwartsmann ${ }^{8}$, Cerli Rocha Gattass ${ }^{9}$, Thereza Quirico-Santos ${ }^{10}$
}

\begin{abstract}
Hospital Universitário Antônio Pedro, Faculdade de Farmácia, Departamento de Biologia Celular e Molecular e Instituto de Biologia da Universidade Federal Fluminense, Niterói, RJ. Serviço de Anatomia Patológica e Serviço de Neurocirurgia do Hospital Estadual Getúlio Vargas, Rio de Janeiro, RJ. Serviço de Oncologia, Faculdade de Medicina da Universidade Federal do Rio Grande do Sul (UFRS), Rio Grande do Sul, RS. Laboratório de Neurogênese e Laboratório de Imunologia Celular, Instituto de Biofísica, Universidade Federal do Rio de Janeiro, Rio de Janeiro, RJ, Brasil.
\end{abstract}

\section{RESUMO}

Introdução: Estudos in vitro mostram que radioterapia e/ou quimioterapia podem ativar as vias de sinalização do receptor do fator de crescimento epidérmico (EGFR) e Ras, aumentando a resistência cruzada das células de glioblastomas multiformes (GBM) ao tratamento. A inibição das atividades de EGFR e Ras através de inibidores tirosinas cinases elimina o antagonismo observado à administração seqüencial destas modalidades terapêuticas, induzindo apoptose nestas células. Em estudo prévio demonstramos que o tratamento com o álcool perílico (AP), inibidor da farnesilação da Ras, induz apoptose em linhagens celulares e células de explante de GBM. Objetivo: No presente estudo investigamos se a regressão parcial observada em GBM recorrente de paciente tratado com administração intranasal de AP é mediada por apoptose. Resultado: Ensaios com TUNEL (deoxynucleotidyl-mediated deoxyuridine triphosphate) e caspase-3 ativada evidenciaram presença de células apoptóticas nas lâminas de GBM tratado. Conclusão: Esses achados sugerem que estratégias adjuvantes visando à inativação das vias de sinalização do EGFR e Ras podem melhorar tanto a eficácia de terapia isolada como de terapia multimodal em gliomas.

\section{PALAVRAS-CHAVE}

Apoptose. Glioblastoma. Quimioterapia. Álcool perílico.

\section{ABSTRACT}

Immunohistochemical detection of apoptotic cells in a recurrent glioblastoma patient treated with intranasal delivery of perillyl alcohol

Background: In vitro studies demonstrated that both radiation and chemotherapy can activate EGFR and Ras signaling pathways, leading to increased cross-resistance to treatment of GBM cell. Inhibition of either EGFR or Ras activity with tytosine kinase inhibitor appears to abrogate the observed antagonism between sequentially administration of these therapeutic modalities inducing apoptosis in these cells. In a previous study, we demonstrated that in vitro treatment with perillyl alcohol (POH), an inhibitor of Ras farnezilation, induced apoptosis in human GBM cell lines and explants. Objective: In the present

1 Professor Adjunto de Neurocirurgia do Hospital Universitário Antônio Pedro da Universidade Federal Fluminense.

2 Doutora do Laboratório de Neurogênese do Instituto de Biofísica da Universidade Federal do Rio de Janeiro.

3 Professora Adjunta da Faculdade de Farmácia da Universidade Federal Fluminense.

4 Neurocirurgião do Hospital Estadual Getúlio Vargas, RJ.

5 Patologista do Hospital Estadual Getúlio Vargas, RJ.

6 Pós-graduando do Laboratório de Neurogênese do Instituto de Biofísica da Universidade Federal do Rio de Janeiro. 7 Professor Titular do Laboratório de Neurogênese do Instituto de Biofísica da Universidade Federal do Rio de Janeiro. 8 Professor Pesquisador do Serviço de Oncologia da Universidade Federal do Rio Grande do Sul (UFRS).

9 Professora Adjunta do Laboratório de Imunologia Celular do Instituto de Biofísica da Universidade Federal do Rio de Janeiro.

10 Professora Titular do Departamento de Biologia Celular e Molecular do Instituto de Biologia da Universidade Federal Fluminense. 
study, we investigated if the partial regression observed in a patient with a recurrent GBM after treatment by intranasal delivery of $\mathrm{POH}$, is mediated by apoptosis. Result: Data from classical histology, terminal deoxynucleotidyl-mediated deoxyuridine triphosphate nick-end labeling (TUNEL) assay, as well as activation of caspase 3, showed increased apoptosis in the treated tumor. Conclusion: These findings suggest that strategies to inactivate EGFR and RAS signaling may be critical to improving not only the efficacy of single-agent therapy but also of multimodal therapy in gliomas.

\section{KEY WORDS}

Apoptose. Glioblastoma. Chemotherapy. Perillyl alcohol.

\section{Introdução}

Importante característica do glioblastoma multiforme (GBM) é sua tendência à recidiva em curto intervalo de tempo devido à resistência dessas células tumorais às terapias convencionais adjuvantes: radioterapia (RT) e quimioterapia (QT). Estudo ${ }^{4}$ mostrou que dano ao ácido desoxirribonucléico (DNA), à administração seqüencial dessas modalidades terapêuticas às células de GBM, pode ativar vias de sinalização da Ras, via receptor do fator de crescimento epidérmico (EGFR), contribuindo para resistência à indução de apoptose. A inibição da Ras e/ou EGFR parece eliminar o antagonismo observado entre RT e QT, realçando indução à apoptose das células de GBM tratadas. Esses achados sugerem que estratégia visando à inativação dessas vias de sinalização pode ser crucial para a eficácia de modalidades terapêuticas combinadas em GBM.

$\mathrm{O}$ álcool perílico (AP), um monoterpeno monocíclico hidroxilado que apresenta efeitos antitumorais em uma variedade de modelos experimentais, possui efeitos antiangiogênicos e pró-apoptóticos, interferindo nas vias de sinalização mediadas pelo oncogene Ras ${ }^{5}$. Estudos pré-clínicos mostram que o AP induz apoptose em células pancreáticas ${ }^{3}$, células leucêmicas $\mathrm{Bcr} / \mathrm{Abl}$ transformadas ${ }^{6}$, células de carcinoma mamário ${ }^{1}$ e linhagens celulares de glioblastoma previamente tratadas com radioterapia ${ }^{10}$. Estudo do nosso grupo mostrou que o AP induz apoptose em linhagens celulares e células de explante de glioblastoma ${ }^{8}$. Desenvolvemos estudo clínico de fase I / II do AP administrado por via inalatória em pacientes com gliomas malignos recidivados após tratamento convencional (estudo aprovado pelo CONEP 9681 no 25000.009267/2004-25). Dentre os pacientes do nosso estudo, dois portadores de GBM foram reoperados após 5 e 6 meses do início do tratamento. Observamos ao exame anatomopatológico do tecido tumoral de GBM recidiado tratado com o AP, a presença de células apoptóticas dispersas (células tumorais), relatadas como áreas com efeito do tratamento com AP e RT.

\section{Material e métodos}

\section{Exame anatomopatológico}

Todo o material cirúrgico obtido do paciente foi remetido ao Serviço de Anatomia Patológica do Hospital Estadual Getúlio Vargas, Rio de Janeiro, e submetido a exame anatomopatológico (GBM inicial e recidivado). No presente estudo, as lâminas histológicas, coradas por hematoxilina e eosina, do GBM inicial e recorrente foram revistas, e blocos de parafina, contendo respectivamente o tecido formalizado do GBM inicial e do recidivado, foram selecionados para os ensaios do TUNEL e da caspase-3 ativada.

\section{Ensaio de TUNEL}

A fragmentação de DNA in situ foi detectada com um kit para TUNEL obtido da Chemicon (Chemicon International Inc., 28820 Single Oak Drive Temecula, CA 92590 - Intergen) de acordo com as instruções do fabricante. Brevemente, corte de 5 micrometros de espessura fora desparafinizado. Os cortes foram tratados com Triton-X100 por $15 \mathrm{mi}$ nutos à temperatura ambiente, lavados 3 vezes com solução tampão fosfato (PBS) e incubados overnight à $37^{\circ} \mathrm{C}$ com enzima TdT (terminal deoxynucleotidyl transferase) e com desoxinucleotídeos marcados com biotina e não marcados. A enzima se liga a terminações 3 '-OH do DNA fragmentado e catalisa a adição de desoxinucleotídeos (marcados e não marcados). A reação foi parada por imersão durante 10 minutos em um tampão específico do kit. Os cortes foram então lavados 3 vezes com PBS (solução tampão fosfato), e incubados com antidigoxigenina conjugada a fluoresceína por 2 horas à temperatura ambiente para reconhecer os desoxinucleotídeos adicionados à fita de DNA fragmentada. 


\section{Resultados}

\section{Caso ilustrativo}

Homem de 49 anos de idade, admitido no Serviço de Neurocirurgia do Hospital Estadual Getúlio Vargas - RJ, em 29 de julho de 2004, com relato de cefaléia, distúrbio de memória, episódio de perda de consciência, sem crise convulsiva. A ressonância nuclear magnética (RNM) de 7 de agosto de 2004 evidenciou volumosa lesão expansiva extra-axial com alto sinal em T1 e sinal heterogêneo em T2, captando de forma moderada e heterogênea o meio de contraste e localizado na região fronto-têmporo-parietal direita. Havia importante edema perilesional determinando compressão das estruturas encefálicas adjacentes, desviando a linha média contralateralmente; suas dimensões foram estabelecidas em $6,0 \mathrm{~cm}$ x 6,4 cm x 5,0 cm (figura 1); a análise espectral mostrou importante aumento do nível de lactato no $\mathrm{TE}=35 \mathrm{~ms}$ no centro da lesão. $\mathrm{O}$ tratamento neurocirúrgico consistiu em ampla craniotomia fronto-têmporo-parietal direita, realizada em 13 de agosto de 2004. Após a cirurgia, o exame neurológico evidenciou hemiparesia esquerda. O laudo anatomopatológico concluiu o diagnóstico de "glioblastoma, grau IV da OMS". Recebeu alta em 29 de agosto de 2004. Iniciou tratamento radioterápico em doses de 5.400 cGy no período de 8 de setembro a 29 de outubro de 2004. No dia 4 de novembro, retornou ao Serviço de Neuro- cirurgia com quadro de sonolência, sem interagir com o meio, hemiplegia esquerda, dificuldade respiratória, impossibilidade de deglutir e desidratado. A tomografia computadorizada (TC) de crânio revelou presença de processo expansivo compatível com recidiva de GBM. Nessa data, iniciamos administração de AP a $0,3 \%$ volume de $8 / 8$ horas, por nebulização. O paciente melhorou o nível de consciência e recebeu alta, após 10 dias, para continuar o tratamento em regime domiciliar. Os exames de sangue, urina e radiografia de tórax, feitos quinzenalmente, mostraram-se dentro dos parâmetros normais. Foram realizadas duas TC de tórax, no primeiro e terceiro meses de tratamento, que foram normais. Não há relato de episódios de cefaléia, vômitos, náuseas, diarréia e eructação durante o tratamento. Os exames de imagem (figura 2) mostram que aparentemente houve redução nas dimensões do volume tumoral sem melhora do edema perilesional. Apesar da melhora do quadro neurológico com o tratamento com AP, o paciente fazia uso de corticosteróides continuamente. No oitavo mês de tratamento, apresentou episódios de vômitos e queda do nível de consciência. A TC de crânio revelou presença de abscesso cerebral. Foi submetido à nova craniotomia para drenagem do abscesso e foi feita ablação do restante do tumor $(1,8 \mathrm{~cm} \times 1,9 \mathrm{~cm})$. O exame anatomopatológico do tecido tumoral mostrou presença de células tumorais e sugeriu a presença de células apoptóticas (figura 2). Ensaios com TUNEL e caspase-3 ativada evidenciaram presença de células apoptóticas nas lâminas de GBM tratado (figura 3).
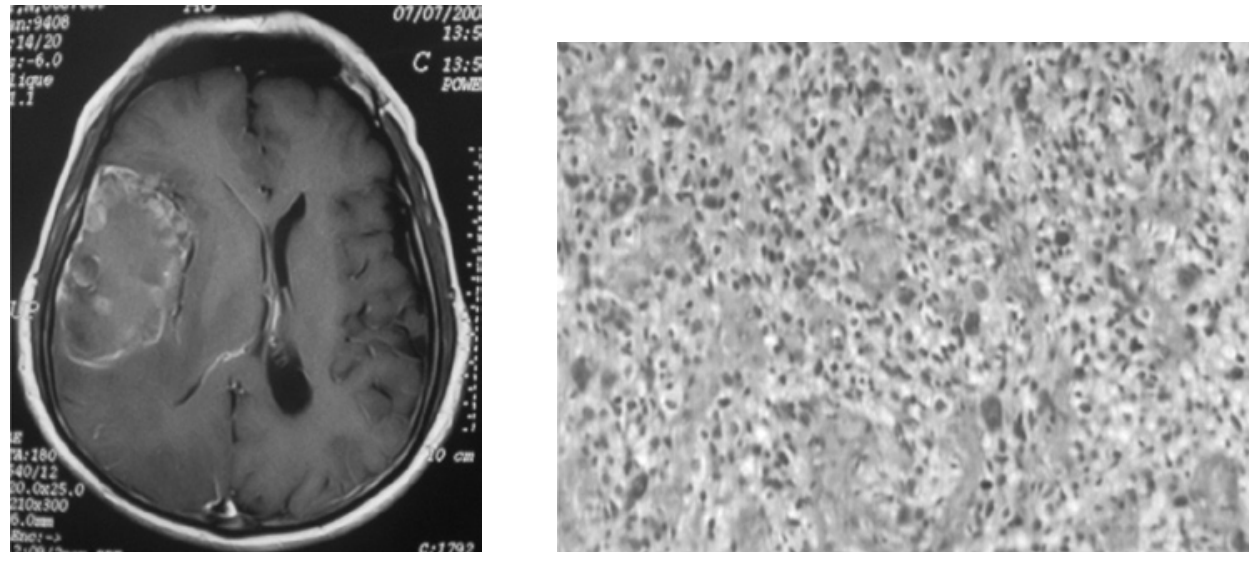

Figura 1 -Detalhe do tumor astrocitico de alto grau com hipercelularidade e pleomorfismo marcante (HE, 100X). 

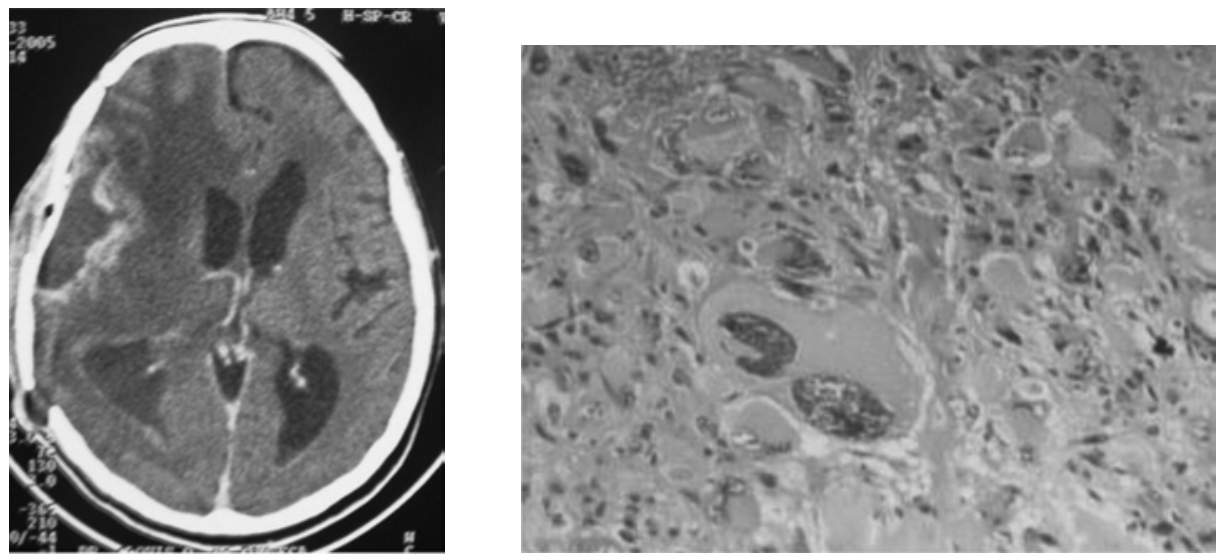

Figura 2 - Efeito do tratamento com AP após radioterapia: células apoptóticas dispersas (células tumorais) em meio às áreas com efeito de radiação $(\mathrm{HE}, 400 \mathrm{X})$.

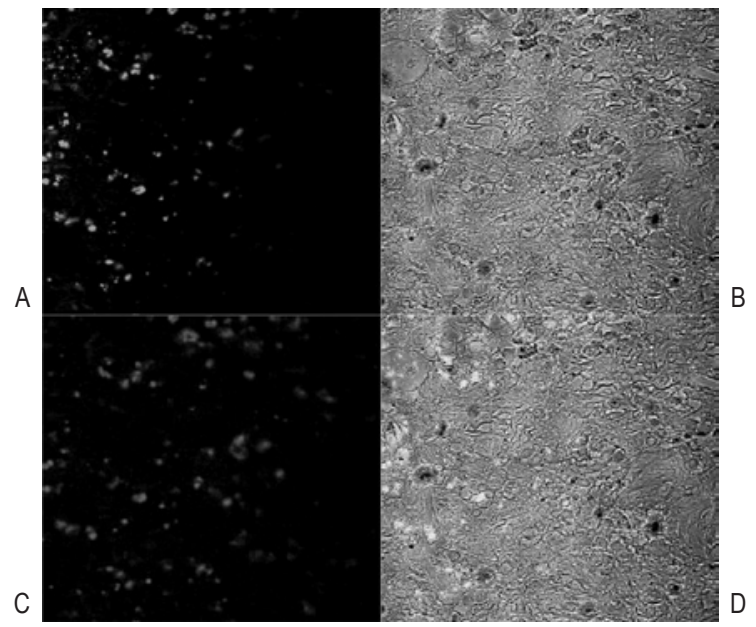

Figura 3 - Ensaio do TUNEL e caspase-3 ativada. A) Sinais de processo apoptótico celular (TUNEL-positivo); B) Controle; C) Imuno-histoquímica - caspase-3 ativada; D) Merge.

\section{Discussão}

A sobrevida média dos pacientes com glioblastomas permanece em torno de 9 a 11 meses, apesar de ablação cirúrgica completa, radioterapia e quimioterapia ${ }^{7}$. Estudos $^{9}$ dos mecanismos moleculares da resistência dos gliomas ao tratamento convencional mostram que radioterapia e quimioterapia podem intensificar a via de sinalização do EGFR ativando a via Ras/MAPK. Estas vias, uma vez ativadas, parecem realçar resistência à indução de apoptose nestes tumores. Pesquisas ${ }^{1}$ demons- tram que terapias anti-EGFR e anti-Ras podem produzir efeitos benéficos quando combinadas às modalidades terapêuticas convencionais. Experimentos mostraram que linhagens de gliomas de alto grau quando expostas durante períodos prolongados com inibidores da p21Ras, sofreram efeitos antiproliferativos e apoptose ${ }^{2}$.

Estudos pré-clínicos mostram que o AP induz apoptose em linhagens celulares de glioblastoma previamente tratadas com radioterapia inibindo a ligação Fas/FasL ${ }^{10}$. Em experimentos pré-clínicos, já havíamos identificado indução de apoptose em linhagens celulares U87 e A172 e células de explantes de GBM tratadas com $\mathrm{AP}^{8}$. Em nosso estudo clínico de fase I / II com o AP por via inalatória, uma observação importante foi a presença de apoptose no tecido tumoral de GBM recidivado que apresentou regressão de volume após o tratamento. O laudo anatomopatológico evidenciou células apoptóticas presentes no tecido tumoral extraído cirurgicamente, o que foi confirmado pelo ensaio com TUNEL e caspase-3. Estes achados sugerem que a regressão tumoral observada após tratamento com o AP por via inalatória foi mediada por apoptose.

Em suma, evidências pré-clínicas e clínicas mostram que a via de sinalização do EGFR e Ras/MAPK intensifica resistência das células tumorais gliais à apoptose, mede a resistência cruzada entre radioterapia e quimioterapia, aumenta o potencial angiogênico, migração celular, acentuando o potencial maligno dos gliomas $^{2,4}$. Portanto é encorajador o estudo farmacológico de drogas que possam inibir essas vias, visando seu uso em terapia multimodal. 
$\mathrm{O}$ relato deste caso sugere potencial benefício do uso do AP como agente quimioterápico para pacientes com GBM recidivado. Entretanto, até que dados ulteriores confirmem nossos achados preliminares, esse agente deveria ser limitado aos casos em que a terapia convencional tenha falhado e aos pacientes arrolados em nossos ensaios clínicos.

\section{Conclusão}

A análise isolada de apenas um caso não nos permite fazer correlação entre os achados descritos e os possíveis efeitos do uso do AP por via inalatória em pacientes com GBM visando seu uso como terapia adjuvante. Contudo, alguns fatores devem ser ressaltados. O efeito terapêutico evidenciado pela redução tumoral e os achados descritos nos exames anatomopatológicos e no ensaio pela técnica do TUNEL.

\section{Agradecimentos}

CNPq, FAPERJ, FINEP/NTQN, Fundação Euclides da Cunha - UFF e AMIL Assistência Médica Internacional.

\section{Referências}

1. ARIAZIEA, SATOMIY, ELLIS MJ, HAAG JD, SHI W, SATTLER CA, GOULD MN: Activation of the transforming growth factor beta signaling pathway and induction of cytostasis and apoptosis in mammary carcinomas treated with the anticancer agent perillyl alcohol. Cancer Res 59:1917-28 1999.

2. BREDEL M, POLLACK IF: The p21-Ras signal transduction pathway and growth regulation in human high-grade gliomas. Brain Res 29:232-49, 1999.
3. BURKE YD, AYOUBI AS, WERNER SR, MCFARLAND BC, HEILMAN DK, RUGGERI BA, CROWELL PL: Effects of the isoprenoids perillyl alcohol and farnesol on apoptosis biomarkers in pancreatic cancer chemoprevention. Anticancer Res 22:3127-34, 2002.

4. CHAKRAVARTI A, CHAKLADAR A, DELANEY MA, LATHAM DE, LOEFFLER JS: The epidermal growth factor receptor pathway mediates resistance to sequential administration of radiation and chemotherapy in primary human glioblastoma cells in a RAS-dependent manner. Cancer Res 62:4307-15, 2002.

5. CHUNG BH, LEE JS, YOUNG CY: Perillyl alcohol inhibits the expression and function of the androgen receptor in human prostate cancer cells. Cancer Lett 236:222-8, 2006.

6. CLARK SS: Perillyl alcohol induces c-Myc-dependent apoptosis in Bcr/Abl-transformed leukemia cells. Oncology 70:13-8, 2006.

7. COUGHLIN C, SCOTT C, LANGER C, COIA L, CURRAN W, RUBIN P: Phase II, two-arm RTOG trial (94-11) of bischloroethyl-nitrosourea plus accelerated hyperfractionated radiotherapy ( 64.0 or $70.4 \mathrm{~Gy}$ ) based on tumor volume $(>20$ or $<$ or $=20 \mathrm{~cm}$ (2), respectively) in the treatment of newlydiagnosed radiosurgery-ineligible glioblastoma multiforme patients. Int J Radiat Oncol Biol Phys 48:1351-8, 2000.

8. FERNANDES J, FONSECA CO, TEIXEIRAA, GATASS CR: Perillyl Alcohol induces apoptosis in human glioblastoma multiforme cells. Oncol Rep 13:943-7, 2005.

9. LI B, YUAN M, KIM IA, CHANG CM, BERNHARD EJ, SHU HK: Mutant epidermal growth factor receptor displays increased signaling through the phosphatidylinositol-3 kinase/AKT pathway and promotes radioresistance in cells of astrocytic origin. Oncogene 23:4594-602, 2004.

10. RAJESH D, STENZEL RA, Howard SP: Perillyl alcohol as a radio/chemosensitizer in malignant gliomas. $\mathrm{J}$ Biol Chem 278:35968-78, 2003.

Original recebido em março de 2007

Aceito para publicação em junho de 2007

\section{Endereço para correspondência}

Clovis Orlando da Fonseca

Rua Alberto Bianchi, 57/101

22795-390 - Rio de Janeiro, RJ

E-mail:clovis.orlando@uol.com.br 